\title{
Entre artesanos y culíes. Trinidad Manuel Pérez (1832-1879), primer autor de las reivindicaciones sociales en el Perú
}

\section{Between artisans and coolies. Trinidad Manuel Perez (1832-1879), a first author of social recognitions in Peru}

Johnny Zevallos ${ }^{1}$

\section{Resumen}

La obra, prácticamente desconocida, del periodista trujillano Trinidad Manuel Pérez ha debido someterse al olvido por parte de la crítica literaria; sin embargo, esta contiene elementos innovadores como la inserción de nuevos sujetos: artesanos, culíes, bandoleros, mujeres lectoras. Nuestra intención es analizar sus piezas dramáticas El emigrado español (1859) y La industria y el poder (1862), así como su novela inconclusa Nurerdín-Kan (1872), primera producción que registra la inmigración de los chinos al Perú a fin de reivindicar las luchas sociales y el protagonismo de las clases menos favorecidas a partir de la incorporación de las renovadoras ideas liberales.

1 Licenciado en Literatura por la Universidad Nacional Mayor de San Marcos. Artículos suyos han aparecido en revistas peruanas e internacionales. Actualmente ejerce la docencia en la Universidad Peruana de Ciencias Aplicadas. Contacto: jotardo.johnny@gmail.com 
Palabras clave: Teatro, novela, siglo XIX, artesanos, culíes

\section{Abstract}

The work, practically unknown, of journalist from Trujillo Trinidad Manuel Pérez has had to submit to oblivion on part of the literary criticism; however, it contains innovative elements such as the insertion of new subjects as artisans, coolies, bandoleros, and female readers. Our intention is to analyze his dramatic pieces El emigrado español (1859) and La industria y el poder (1862), as well as his unfinished novel Nurerdin-Kan (1872), first production that records the immigration of the Chinese people to Peru in order to claim the social struggles and the participation of peoples from the incorporation of innovative liberalism.

Keywords: Theater, novel, nineteenth century, artisans, coolies

$$
* * *
$$

\section{Introducción}

Hacia mediados del siglo XIX el teatro y la poesía constituían sin duda los géneros literarios más consumidos por el público limeño. Gracias a la incursión de Felipe Pardo y Aliaga y de Manuel Ascensio Segura, las producciones dramáticas habían abandonado el germen neoclásico y corte-

114 sano para girar hacia un modelo costumbrista. Sin embargo, la ficción narrativa empezaba a tener auge a partir de la década de 1840, pues ya existía un significativo número de narraciones importantes; de hecho, el folletín se convertirá en la vía de circulación de novelas y producciones ficcionales breves. Los mundos representados en estos textos recogían principalmente las costumbres y modos de interacción entre criollos, mestizos, indígenas y afrodescendientes, así como 
las posibilidades de proyectos nacionales que empezaban a surgir en el Perú anterior a la guerra del Pacífico.

Estos proyectos nacionales representados ficcionalmente no incluían, como era de suponerse, las ingentes oleadas migratorias que comenzaron a circular por tierras peruanas. La asimilación de italianos, alemanes y asiáticos no estaba contemplada en estas producciones narrativas, sino como personajes alternos, a pesar de que la presencia de estos últimos era cada vez más notoria. No hubo, por lo tanto, una preocupación por representar a los jornaleros chinos que arribaron a nuestras costas a partir de la segunda mitad del siglo XIX a excepción de los poemas de Juan de Arona y la novela anónima Nurerdín-Kan.

Nurerdin-Kan apareció sin firma y a manera de folletín en el semanario El Correo del Perú, en la edición correspondiente al año 1872, y ocupó veintiséis números entre los meses de enero y julio. Este semanario era dirigido por el periodista y dramaturgo trujillano Trinidad Manuel Pérez, integrante de la Bohemia limeña de mediados del siglo XIX y conspicuo admirador de la novedosa tradición literaria de la capital. Por sus páginas aparecieron los más importantes poetas, dramaturgos y periodistas peruanos y latinoamericanos de la época los siguientes: Ricardo Palma, Manuel González Prada, Teresa González de Fanning, Juana Manuela Gorriti, Mariano Amézaga, Luis B. Cisneros, Constantino Carrasco, Francisco de Paula González Vigil, Francisco García Calderón, Eugenio Larrabure y Unanue, Francisco J. Mariátegui, Juan A. Ribeyro, Carolina Freyre de Jaimes y la española María del Pilar Sinués de Marco, entre otros.

Pretendemos reforzar la tesis de Alberto Tauro, quien sostuvo que Trinidad Manuel Pérez, director del semanario El 
Correo del Perú, fue, en efecto, el autor de la novela, hecho que el eminente crítico peruano no detalla. Para dicho propósito, compararemos el estilo y los diálogos de la novela con dos producciones teatrales del periodista norteño: El emigrado español (1859) y La industria y el poder (1862). Asimismo, buscamos enfatizar el papel de Pérez como primer autor que aspiraba representar a los sectores populares y su afán político por ser reconocidos como agentes sociales en una república que empezaba a nacer.

\section{Trinidad Manuel Pérez, una vida de ficción}

Trinidad Manuel Pérez nació en Trujillo el 30 de mayo 1832 y llegó a Lima en 1851 cuando aún era «un muchachote llano como camino real, siempre alegre, y bravo sin fanfarronería. Su color moreno, y su negro y poblado bigote; su alta estatura; el cabal desarrollo de su cuerpo y su fuerza muscular, imprimían en su persona algo del tipo árabe» (1961: 13111312). Años más tarde viaja a Europa, «de donde regresó trayendo las prensas y tipos de la mejor imprenta que, hasta 1871, se hubiera conocido en Lima» (Palma 1961: 1312). Es en ese año que funda, junto a su hermano Isidro Mariano, el reconocido semanario El Correo del Perú (en adelante: ECP), cuya pulcra edición y cuidada presentación le valieron sendos premios «en las exposiciones de París, Filadelfia, Ámsterdam,

116 Lima y Santiago» (1871: 1312). El tradicionista asevera que los hermanos Pérez, sobre todo Trinidad Manuel, se preocuparon por que ECP cuente con los mejores periodistas y escritores de la época; de esta forma, colaboraron en sus páginas Ricardo Palma², Manuel González Prada, Francisco de

2 Palma publicó la segunda serie de sus Tradiciones peruanas en ECP en 1872. Allí figuraron producciones como «A la cárcel todo Cristo!», 
Paula González Vigil, Federico Flores Galindo, Juan de Arona, Pedro Antonio Varela, las escritoras Carolina Freyre de Jaimes, Teresa González de Fanning, Mercedes Cabello de Carbonera, Clorinda Matto de Turner, entre otros. No obstante, el semanario contó también con el aporte de notables dibujantes y artistas gráficos que le brindaron una brillante presentación al periódico y elevaron su trascendencia como medio de comunicación en las veladas y discusiones literarias de la época; entre ellos, A. Dreux, L. Dumont y J. Gaildreau (Zanutelli 2006: 288). En 1878 Trinidad Manuel abandona sus labores en ECP para viajar a la provincia de Chota, pues había adquirido la hacienda San Antonio de Chancay, propiedad de su suegro que había fallecido pocos días antes (Palma 1961: 1312). Don Ricardo lamenta la decisión del periodista trujillano, pues las personas que permanecieron a cargo del semanario no pudieron darle continuidad, lo que significó la desaparición de uno de los periódicos más prestigiosos del Perú decimonónico. Palma añade que, durante su estancia en la hacienda, Pérez fue asaltado y asesinado por un grupo de bandoleros el 9 de septiembre de 1879 (1961: 1312). Escribió dos obras dramáticas, La industria y el poder y El emigrado español, y una novela, Nurerdín-Kan.

\section{La representación de los artesanos y de los culíes}

La industria y el poder fue publicada originalmente en 1862 y contó con dos ediciones más; la última, aparecida en 1875. El investigador estadounidense Paul Gootenberg, uno de los mejores historiadores económicos sobre el Perú decimonónico, asegura que Trinidad Manuel Pérez se involucró con

«Con días y ollas venceremos», «Un virrey hereje y un capellán bellaco», «Beba, padre, que le da la vida!», entre otros. 
la Sociedad de Artesanos de Lima y Callao y hasta le dedicó su mayor pieza teatral: La industria y el poder, obra que exalta a la clase trabajadora y que muestra a los artesanos como personajes literarios (Gootenberg 1998: 212-213). El historiador añade: «la obra encarna formalmente el viejo 'orgullo artesano' de los gremios, no obstante estar influida por el drama social europeo contemporáneo. A pesar de no ser nada atractiva para los críticos limeños, La industria y el poder tuvo dos ediciones más y complació a públicos simpatizantes hasta bien entrada la década de 1870» (1998: 212). Íñigo García Bryce sostiene que esta pieza teatral ayudó a legitimar la asociación de artesanos en Lima y consolidó su presencia entre los liberales (2008: 168). Sin embargo, Jorge Basadre, en su monumental Historia de la República del Perú, sostiene que el interés en La industria y el poder estará dirigido hacia los historiadores sociales antes que los críticos literarios, por cuanto esta pieza dramática «carece de valor estético y teatral. Su trama peca de inverosímil y forzada» (1983: IV, 385). Nuestro interés se centra en validar el propósito de Pérez de incorporar a este sector dentro de la producción discursiva criolla en cuanto formularía un primer paso para la construcción de una nación letrada inclusiva. Tampoco pretendemos soslayar el valor estético que debe cumplir toda obra teatral para solidificar su papel integrador, sino priorizar la participación de los sectores subalternos en cuanto creadores lite-

118 rarios en sí mismos.

La industria y el poder es una pieza construida a partir de modelos costumbristas que, si bien no llega a igualar los dramas de Felipe Pardo y Manuel Ascensio Segura, contiene ya aires románticos reconocibles como la mujer criolla que aspira a romper los límites sociales para unirse con un joven artesano. Esta pieza representa uno de los primeros recursos 
literarios en el que se apela al sentimentalismo doméstico, propio del discurso liberal y de quienes aspiraban a fundar una sociedad basada en modelos progresistas. El título de la obra refiere indudablemente a la dicotomía «industria», es decir, artesanos u obreros y «poder», esto es, poder político. Por consiguiente, el texto constituye un alegato de la conciencia política que poseían ya lo artesanos en el siglo XIX. Es posible advertir entonces un afán por enunciar una obra teatral inspirada en los patrones democráticos respecto del artesanado, pues intentaba «dar voz a las preocupaciones de este grupo y presentar sus demandas» (García Bryce 2008: 221). De esta manera, Trinidad Manuel buscaba insertar a este pujante sector socioeconómico dentro de la ciudadanía como una forma de garantizar su fortaleza como gremio o grupo socioeconómico. Por ejemplo, en la dedicatoria que Trinidad Manuel dirige a la Sociedad de Artesanos de Lima y Callao en su pieza teatral, considera necesario que los artesanos deban «asociarse para fomentar la industria nacional, y auxiliarse mútuamente para garantizar el trabajo, y defenderse contra la inaccion que trae la miseria» (Pérez 1875: 5). Además, Íñigo García-Bryce señala que la educación se convirtió en el principal vehículo que hallaron los articulistas de los periódicos a favor de la clase obrera para convertir a los artesanos en ciudadanos y, sin duda, esta obra del dramaturgo trujillano fue una pieza clave en esa tarea (2008: 221). Como indica E.P. Thompson, al estudiar el surgimiento de la clase obrera británica, realidad que no se alejaría mucho de la de otras regiones menos desarrolladas:

Una vez aprendidas las técnicas elementales de la lectura y la escritura, los peones, artesanos, tenderos, oficinistas y maestros de escuela procedían a instruirse, ya fuese individualmente o en grupos (...). 
De este modo los obreros se formaron una imagen de la organización de la sociedad, a partir de su propia experiencia y con la ayuda de una educación desigual y conseguida a duras penas: una imagen de la sociedad que era, ante todo, política (2012: 762).

La industria y el poder se centra en la historia de amor entre Pablo y Edelmira, dos jóvenes limeños que luchan por su sentimiento amoroso frente a los prejuicios establecidos por una estructura social oligárquica que rechazaba la presencia de los trabajadores dentro del espacio hegemónico criollo. Pablo, un joven artesano limeño que labora en el taller de don Lorenzo, su padre, se enamora perdidamente de Edelmira, una señorita capitalina de alcurnia que había recibido la crianza y los cuidados de Elena, a quien se le había entregado la niña tras la muerte de su madre. Sin embargo, el amor de Edelmira también era deseado por Pío, hermano de Elena, quien estaba más interesado en la fortuna de la dama que en su afecto. Pablo goza del aprecio y la protección de Elena, quien no duda en aprobar la relación sentimental entre los dos jóvenes y, por el contrario, condena los intereses de su hermano. Por ello, a fin de concretar sus planes, Pío y su padre, Fernando Aguilar, traman una calumnia contra Pablo, a quien acusan de ser el amante de Elena, para lo cual deciden escribir una esquela en la que la mujer espera al joven en su habitación. Por fortuna, Edelmira se anticipa a Elena e ingresa a su habitación donde Pablo le explica las razones de por qué se hallaba invitado en la estancia de su protectora. Pero, aunque Pío consigue expandir el rumor de que Elena y Pablo eran amantes, aquella decide comunicarle a Alberto, su esposo y ministro plenipotenciario, sobre la conspiración armada en su contra. El ministro decide entonces recluir a Pablo en una pequeña prisión; sin embargo, don Lorenzo consigue 
una cita con Alberto a quien explica la inocencia de su hijo frente a tan cruel conspiración. Pero al oír la voz del anciano, el ministro reconoce a su progenitor, a quien no veía desde sus primeros años. Pablo, en cuanto representante del artesanado limeño, rompe las barreras entre la aristocracia limeña y el sector popular al pretender unirse a Edelmira. Por ello, Alberto cree ver en su hermano a sí mismo, pues, al igual que él, también es de origen humilde. Finalmente, el plan de Pío es descubierto, y Edelmira y Pablo consiguen unir sus vidas, mientras que Alberto y Elena aprueban el matrimonio de los jóvenes amantes.

La obra constituye una construcción alegórica de la lucha del artesanado por reconocer sus derechos como ciudadanos y su inserción en el espacio político y social de la Lima del siglo XIX. La ideología liberal que expresa Trinidad Manuel Pérez se inclina por construir una comunidad centrada en valores como la honestidad y el trabajo, propios de una sociedad inspirada en su propia conciencia de pertenencia a una clase social. Así, el autor encuentra una dicotomía bastante diferenciada entre artesanos «honestos» y aristócratas «deshonestos», pues considera relevante concientizar el ascenso social como una manera de superar a la sociedad oligárquica, modelada por patrones injustos e indecentes. Las figuras de Pablo, Lorenzo y Alberto están regidas por categorías morales que determinan su representatividad a partir de paradigmas sociales establecidos por una ética decimonónica: bondad, honestidad, laboriosidad. Por el contrario, se castiga a la oligarquía criolla, en la medida de que se le considera responsable de la injusticia y el padecimiento del artesanado limeño. Es posible advertir, en consecuencia, que Pío y Fernando presentan códigos opuestos a los héroes de la obra de Pérez, puesto que se les antepone patrones contrarios a una moral cristiana preestablecida. 
Así, en Pío y su padre puede advertirse el deseo de impedir el ascenso de los obreros limeños al considerarlos un peligro para la aristocracia criolla, comprometida a preservar su poder hegemónico a toda costa.

Ya en esta pieza teatral se exponen, por consiguiente, los criterios que permitieron al autor identificarse con los sectores menos favorecidos por la mirada hegemónica criolla y su interés por representarlos. En ese sentido, puede considerarse entonces un antecedente de la novela Nurerdín-Kan, por cuanto es posible establecer criterios de comparación entre los dos materiales literarios. Si bien la temática de ambas es distinta, existen algunos aspectos que las emparientan. En primer lugar, el deseo de representar a una sociedad oligárquica deseosa de explotar y condenar a los sectores menos favorecidos, a partir de códigos sociales reconocibles: el empresario criollo truhán e interesado en impedir el ascenso del artesano diligente y honesto en La industria y el poder, y, por otro lado, el hacendado opresor y malicioso en Nurerdin-Kan, sugestionado con castigar y amedrentar a los asiáticos. Asimismo, se observa el afán por parte del autor de conseguir un discurso ficcional cercano a la realidad a través de situaciones cotidianas que reproducen el mundo de los personajes representados en estas producciones. Así, por ejemplo, en la pieza teatral advertimos escenas de debate político, en

122 el que se menciona el interés de los artesanos por ser reconocidos como un grupo social capaz de reclamar sus derechos como ciudadanos e intervenir en política, mientras que en la novela el autor plasma el lamento de los chinos en los barcos y las haciendas azucareras y algodoneras. En segundo lugar, existen estrategias discursivas que emparientan a ambas producciones, ya que es posible descubrir parlamentos con características muy similares. De esta manera, se observa que 
el diálogo entre Alberto, joven de origen humilde que aspira alcanzar un mayor sitial en la sociedad burguesa limeña; su padre, Lorenzo, viejo artesano de la ciudad; y los ricos hacendados Pío y Fernando, pretende reconocer el valor del sector obrero decimonónico.

\section{Alberto}

—Deténgase usted. - Ya bastante he cumplido con las exigencias de la sociedad, para permitir que la etiqueta me prive la inmensa dicha que viene á proporcionarme este momento. Yo, señores, no he aceptado una magistratura para satisfacer mezquinas pasiones, y halagar la pueril vanidad de los que me rodean, no: la he aceptado para realizar los sueños dorados de mi juventud, ser útil á mi patria, y enaltecer la clase industriosa, en cuyo seno nació mi padre. Sí, no me avergüenzo de confesarlo: soy hijo del pueblo. El compás de un obrero midió las dimensiones de mi cuna, y ese obrero era mi padre; tengo orgullo de no haber comprado hasta ese objeto, que sin los artesanos, no habria mecido el sueño de esos hijos de la opulencia y de la ociosidad.

\section{Don Fernando}

- (á Pio). ¿Qué querrá decir con todo esto?

$$
\text { Pío }
$$

-Lo ignoro.

\section{Alberto}

-Venga usted, anciano, y diga usted á estas gentes, quién es usted (Andrés y Pablo aparecen en el fondo).

Lorenzo

-Yo..... no soy mas que un pobre artesano (Pérez 1875: 56-57). 
Con este pasaje nos adentramos al aspecto central de la obra: la reivindicación del sector obrero y su presencia cada vez más clara en su intención por reclamar un espacio de la nueva sociedad limeña. La revelación de Alberto constituye el valor fundamental de una aspiración social que trajeron consigo las ideas liberales: el surgimiento de nuevos personajes en el escenario político y cultural. El liberalismo permitió que desaparecieran paulatinamente algunos muros sociales heredados del antiguo régimen y se diera oportunidad a nuevos sujetos rivalizar con los otrora dueños de haciendas. Al denominarlos «hijos de la opulencia y de la ociosidad», Alberto busca no solo desagraviar el honor de su padre, sino, sobre todo, proponer que como artesano tiene los mismos derechos que un criollo de abolengo. La referencia a la ociosidad como característica de los hacendados en cuanto a seres parasitarios del trabajo de los peones y jornaleros reclama de por sí un primer acercamiento a las luchas sociales que demandaban los agentes económicos populares. Además, la inserción del término «clase industriosa» legitima el espacio político de los obreros como sujetos consecuentes de su poder y anuncia el valor que muchos teóricos sociales añadirán a la población obrera, y su conciencia de clase social y económica (Thompson 2012: 761).

\section{Narrativa y teatro: bases de una reivindicación social}

Un ejemplo similar al de la pieza teatral La industria y el poder lo hallamos en la novela Nurerdín-Kan. Nurerdín-Kan, príncipe de la India, decide abandonar su patria y trasladarse al puerto chino de Macao, entonces colonia portuguesa. Las facciones y vestimenta del personaje llamaron la atención en el bergantín Doria, comandado por el capitán Giacomo Castelli, inhumano navegante italiano especializado en la captura 
de asiáticos. El iracundo capitán, aficionado al lucro, lacónico y autoritario, se niega en un primer momento a embarcar a Nurerdín, pero, tras dialogar con el armador del barco y examinar con detenimiento sus condiciones físicas, cree necesario ofrecerlo al consignatario. El trayecto fue sumamente hostil para el indio y los chinos, quienes eran transportados en una estrecha bodega, húmeda y pestilente. Sin embargo, el ambiente dentro de la bodega comenzó a empeorar ante la escasez de alimentos y el contagio de la peste. De pronto, revólver en mano, el capitán decidió que los culíes debían regresar a la bodega y apuntó sobre la cabeza de uno de los chinos. Nurerdín creyó conveniente salir en defensa de los asiáticos y se enfrentó a Castelli. Los días siguientes transcurrieron con serenidad hasta el arribo del Doria en el puerto del Callao.

Don Remigio Trueba, propietario de uno de los latifundios más prósperos de la costa norte del Perú: la hacienda Las Palmas, había realizado los trámites respectivos para importar trescientos culíes desde la lejana China, debido a la falta de brazos en la agricultura nacional. Acompañado de Rosa, su hija, la aya de esta, Belica, y José, pequeño mulato que trabaja en la casa-hacienda, efectuó el camino sin ninguna complejidad en un vagón de primera clase. El desembarco del Doria se produjo sin contratiempos. Sin embargo, con motivo de las celebraciones del 9 de diciembre, fecha en que se conmemoraba la consolidación definitiva de la independencia del Perú, los asiáticos despertaron la hilaridad de los transeúntes, quienes no cesaban de emitir estruendosas carcajadas. Al producirse el estallido de los fuegos el gentío se volcó hacia la plaza ahuyentando a los culíes. Animado por los sentimientos de compasión de Rosa, el hacendado inglés y vecino de Las Palmas, míster Hudson, decide ir hacia la 
plaza en su intención de ayudar a los asiáticos y cautivar a Rosa; sin embargo, su lentitud permite a Nurerdín aparecer en escena y reducir a un importante número de mataperros.

En enero de 1861 la comitiva tomó por fin camino a la hacienda. Cerca de Las Palmas un grupo de bandoleros inició su ataque contra los chinos. Las piedras lograron herir mortalmente a uno de los asiáticos, a la vez que los cinco restantes estaban estremecidos. Don Remigio ordenó entonces cubrir con piedras el cadáver y retomaron los pocos pasos que restaban hacia su destino final. Se organizó entonces una pequeña recepción en la casa de los Trueba, a cargo del mayordomo Justo García, para recibir a sus propietarios y el nuevo destino de la hacienda tras la llegada de los asiáticos.

En una de las escenas del relato, el narrador penetra en la privacidad de Rosa Trueba, hija del propietario de la hacienda Las Palmas, y de su amiga Elena para que el lector pueda identificar los sentimientos más profundos de una de ellas.

— ¿Nunca has pensado en el amor?

— ¿Y por qué me lo preguntas, Elena?

-Porque los que tú tienes, dicen, que son los síntomas de esa enfermedad.

—Amor! repitió Rosa pensativa... ¿y á quién podria yo amar?

— iVaya! eso no falta. Hay jóvenes tan buenos mozos.

- Y tan elegantes, añadió nuestra heroina con un tono de indefinible ironía.

— iCierto! Y luego, ¡cuántos no te harian la corte, ingrata!

Rosa guardó silencio. 
Elena, que era una criatura adorable y que amaba sinceramente á su amiga cuando la mayor parte de las mujeres la envidaban y la aborrecian, prosiguió con ingenuidad.

—Mira, hija; yo sola sé de tres jóvenes que se mueren por tí.

—Veamos quienes son.

Elena con el mas gracioso ademan se inclinó entonces al oido de su amiga, y pronunció tres nombres.

Rosa erguió su cabeza sonriéndose con el mas profundo desdén.

— ¿Quieres que te hable con franqueza, Elena? dijo.

-Ya te oigo.

—Pues bien; es cierto que en el baile, en el teatro, en el paseo, en todas partes me encuentro siempre rodeada de muchos jóvenes que me hablan de amor, que procuran agradarme, que me hacen mil juramentos, que me cortejan (Pérez 1872: 126).

Es necesario tener en cuenta que los espacios de socialización cumplían una función importante para el cortejo, pero también para la pérdida de las buenas costumbres, lo cual implicaba, ciertamente, la censura de la participación de las mujeres en este tipo de eventos. Un punto a destacar es que no se condena la presencia del sujeto femenino; por el contrario, el autor estimula la interacción en los teatros y los bailes como una manera de integrar a la mujer limeña junto a sus pares europeas. De esta manera, el espacio de socialización se convierte en un medio de libertad de las mujeres, lo que estimularía un recurso literario creado por un mismo autor, ya que coinciden en la percepción de cómo debía desenvolverse el sujeto femenino en el mundo doméstico. Sin duda la mirada de lo femenino en Trinidad Manuel Pérez 
tiene puntos en común, pues el autor trujillano consideraba necesaria la educación de la mujer en la medida de que significaba una vía para la modernización del país. Así, en el semanario El Correo del Perú se difundieron sendos artículos que abogaban por la activa participación de las mujeres en el quehacer intelectual y literario, así como la educación y los nuevos hábitos femeninos.

Otra de sus piezas teatrales, El emigrado español, contiene algunas pistas que nos permitirán descubrir estrategias literarias análogas a las de Nurerdin-Kan y desarrolla una temática que se aproxima a los ideales políticos de los liberales criollos. En efecto, esta obra dramática tiene como temática el arribo de un ciudadano español, don Fernando Castelar, a la ciudad de Lima a fin de legitimar los amoríos entre Federico y Constanza, su hija. Sin embargo, Constanza debe enfrentar las pretensiones de don Pedro, malvado truhán que desea a toda costa casarse con la joven con el propósito de adquirir sus dotes antes que expresar un sentimiento veraz. Sin embargo, don Pedro tiene en Federico a un rival duro de vencer, por lo que decide asesinar a Claudio, hermano menor de don Fernando, y culpar al joven del homicidio. La noticia de la muerte de su hermano obliga al caballero español a arribar a Lima a fin de resolver el crimen y castigar al asesino. Cuando está por suceder la boda entre Constanza y don Pedro, Federico, Carolina y Gustavo, estos últimos, amigos de

128 Constanza, irrumpen la ceremonia para prender a don Pedro. Afortunadamente, los jóvenes contaban con las pruebas para demostrar la inocencia de Federico y culpar al anciano. Por último, don Fernando saluda la relación entre el joven y su hija, con lo que se pone fin a la pieza teatral.

Es interesante observar que, en El emigrado español y NurerdinKan, la heroína es hija de un caballero peninsular y está ena- 
morada del héroe, quien pertenece al proletariado limeño o está asociado a los sectores desprotegidos. En efecto, Federico es hijo de don Diego y doña Luisa, pareja de ancianos cuya condición social se asemeja a la de los artesanos de la Lima decimonónica, mientras que si bien Nurerdín-Kan es un príncipe indio se compadece y auxilia a los culíes o esclavos chinos. Asimismo, ambas jóvenes están asociadas a una enorme dote y son poseedoras de una belleza envidiable, además de ser pretendidas por hombres maduros cuya intención es su herencia económica. De esta forma, podemos observar ciertas similitudes entre don Pedro y míster Hudson, pues mientras el primero es un anciano despiadado, capaz de asesinar y culpar a su rival de dicho homicidio, el segundo no duda en castigar terriblemente a los culíes que laboran en su hacienda. Ambos son descritos como seres patéticos y malvados, lo que incentiva el odio hacia los grupos dominantes extranjeros, por cuanto se les cataloga como interesados por atormentar a los sectores marginados y arrebatar la herencia de sus anheladas y jóvenes cónyuges. Finalmente, un aspecto interesante es la representación misma de Fernando Castelar y Remigio Trueba, pues mientras el primero es descrito como bondadoso y justiciero, el segundo es representado como opresor y cruel contra los culíes, a quienes no duda en castigar ferozmente. Ambos son españoles y padres de hermosas doncellas, sobre quienes recae la conducción del espacio económico del mundo ficcional descrito en estas obras, ya que se considera que el ciudadano peninsular cuenta con la legitimación del pasado virreinal y, por ende, debe ser erradicado. Sin embargo, al primero se le presenta como extraño al Perú, alguien alejado a la realidad peruana y sus problemas, mientras que al segundo se le ve inmerso en el problema agrario nacional y en la importación de chinos en las haciendas. 
Otro aspecto a destacar en esta pieza dramática es el uso de los parlamentos, los cuales tienen también algunas similitudes con Nurerdín-Kan. Los diálogos en esta obra dramática dan cuenta de algunas escenas que la acercan a la novela, de manera que facilita la proximidad en los protagonistas. Así, por ejemplo, cuando don Pedro es sorprendido por Gustavo y Carolina, al hallársele culpable de la muerte de Federico Montemar, se observa cierta ironía, ya que este es ridiculizado ante los demás personajes y, por ende, los espectadores.

D. PE.- Y tú: ¿como puedes probar lo que tan indignamente me afrontan, cuando eres criminal?

Gus._- (por dentro) ¡Miente! [saliendo y presentando un papel]

D. PE.- ¿Quién se atreve á desmentirme?

Gus.- Yó: que acabo de arrancar al moribundo Claudio, dependiente y cómplice de usted, esta declaracion que ha legalizado un escribano en este instante, junto á su lecho de muerte.

D.PE.- ¡Calle usted!

Gus._- ¡Oh! no: mucho he sufrido para callar ahora, además la mano de Dios pesa sobre usted; pues Claudio muere en...ve...ne...

D.PE._ ¡Calle usted! ¡Calle usted! (Pérez 1859: 52).

Una escena similar es posible hallarla en Nurerdin-Kan, al constatar el escarnio al que es expuesto míster Hudson, al negarse a intervenir durante la golpiza a la que son sometidos los culíes en la plaza de armas de Lima. Rosa Trueba, la joven de la que está perdidamente enamorado, así como el padre de esta, don Remigio, y las personas más allegadas a 
ella no dudan en mofarse de la cobardía del hacendado inglés y, por el contrario, reconocen la valentía de Nurerdín. Los acontecimientos que se relatan tienden, indudablemente, a sublimar las categorías positivas del joven indio, en la medida de que no solo se apiada de los asiáticos, sino que físicamente está más preparado para un enfrentamiento, por cuanto su fortaleza física se debe a los poderes sobrenaturales que le otorga la tradición mitológica de la India y, por el contrario, se subestima al terrateniente británico, ya que se ironiza su debilidad ante la muchedumbre plebeya.

El inglés venia mohino.

—¿Qué ha sucedido, mister? le preguntó $\mathrm{D}^{\mathrm{a}}$. Belica.

- Viendo que los celadores no me hacian caso cuando les suplicaba que fuesen á protejer á los chinos, fuí á buscar á un inspector amigo para que les hiciese obedecer. Volvia, cuando ya he visto que se retiran tranquilamente guiados por José.

—Llegó V. tarde, observó irónicamente Rosa.

Mister Hudson habría querido que se abriese la tierra para él á esta pregunta.

—Gracias, sin embargo, continuó la jóven en el mismo tono.

- Ay! si lo hubiera visto con qué valor, con qué decision se puso á defender á sus compañeros, dijo Elena.

—¿Quién? preguntó Hudson.

-Nur... nu-Kan.

—Nurerdin-Kan, rectificó la hija de D. Remigio. 
- ¿El indio?

-Sí, sí, continuó Rosa con entusiasmo. Ese indio que es todo un tipo de las antiguas leyendas.

Mr. Hudson se mordió los lábios (Pérez 1872: 182).

Ambos fragmentos inciden en elevar las categorías negativas de los rivales del protagonista, pues son estos quienes reivindican su propio honor frente a aquellos. En el primer caso, se advierte el acto de culpar a un inocente de la muerte de una persona honorable y atributos benévolos, factor que se opone a la poca astucia de don Pedro, pues este ha dejado evidencia de su culpabilidad, hecho que despierta ironía y la burla. Además, cabe indicar que este acto frustrado se relaciona con el interés del autor por ridiculizar a la clase hegemónica y demandar la argucia y la valentía de los sectores menos privilegiados, lo que se entiende como la sublimación de un joven obrero a través de su capacidad para desenmascarar a su oponente. En el segundo caso, se aprecia la extraordinaria presencia de Nurerdín-Kan y su destreza para combatir a los opresores de los asiáticos, que se asocian a los sectores populares marginales. Es interesante advertir que, en la obra de Trinidad Manuel Pérez, los mataperros se oponen a los obreros, porque los primeros contienen códigos negativos (asaltantes o aprendices de bandoleros), mien-

132 tras que los segundos representan a los sectores emergentes de la ciudad (empeñosos, luchadores y perseverantes en sus talleres de trabajo). El autor no duda en describir parte de la mitología india para legitimar el origen divino del joven y, de esta manera, ahuyentar a los mataperros. Aunado a ello, es interesante destacar la mirada y la posición de Nurerdín frente a los otros personajes, pues, si bien se le asocia con los chinos, se exalta su origen noble para diferenciarlo de estos. De 
allí que se oponga a los atributos negativos de míster Hudson, a quien el autor describe como interesado en la dote de Rosa Trueba y férreo castigador de los asiáticos. Tanto la joven heredera como los débiles culíes representan, efectivamente, el poder económico descrito en la novela, pues el matrimonio con esta significa la ingente herencia de su vecino y los segundos, los brazos para labrar la tierra. Esta vil aspiración por pretender a ambos sujetos lo describe como incapaz de hacer justicia y luchar por los sectores menos privilegiados por la sociedad limeña decimonónica. Asimismo, debe observarse que tanto don Pedro como míster Hudson pretenden la mano de la joven amada de sus respectivos rivales, Federico y Nurerdín-Kan, lo cual encierra aún más los vínculos cercanos entre ambas producciones discursivas.

\section{Conclusiones}

Existen indicios que nos han permitido establecer con claridad que Trinidad Manuel Pérez fue el autor de la novela Nurerdín-Kan. De esta manera, a partir de los dramas que escribió, La industria y el poder y El emigrado español, es posible determinar algunas pistas que permiten deducir su autoría. La semejanza entre las piezas teatrales y la novela en estudio radica en la construcción alegórica de la lucha de los artesanos y su inserción en la esfera política y social. Asimismo, es posible advertir en los diálogos que el espacio de socialización cumple un papel importante para la libertad de las mujeres. El protagonista, la joven heroína y el pretendiente comparten intereses y estados cronológicos muy similares.

El autor describe con minuciosidad las condiciones que debieron enfrentar los culíes desde su traslado desde el puerto chino de Macao hasta las haciendas. No deja de sorpren- 
der su intención por conseguir un discurso testimonial de la presencia china en nuestro país a partir de una posición objetiva que coincidiera con la descripción misma de los hechos. La síntesis histórica expuesta en el primer capítulo y la construcción imaginada en torno a los chinos permiten colegir que Trinidad Manuel Pérez conocía con detalle los sucesos que acompañaron a los asiáticos y su intención por desmoronar la condición oprobiosa en que estos hechos se realizaron.

Finalmente, Trinidad Manuel Pérez se constituiría en el primer autor en reivindicar las luchas sociales y el protagonismo de las clases menos favorecidas: el artesanado, los jornaleros asiáticos y los bandoleros. Se trata, entonces, de un escritor pionero que halló en la literatura un arma para consolidar el papel de los agentes populares que buscaban una voz en nuestras letras.

Recibido: 15 de mayo del 2017

Aprobado: 12 de agosto del 2017

\section{Bibliografía}

BASADRE, Jorge

1983 Historia de la República del Perú (1822-1933). 11 tomos. Lima: Editorial Universitaria.

GARCÍA-BRYCE, Íñigo

2008 República con ciudadanos. Los artesanos de Lima, 18211879. Lima: Instituto de Estudios Peruanos.

GOOTENBERG, Paul

1998

Imaginar el desarrollo. Las ideas económicas en el Perú postcolonial. Lima: Instituto de Estudios Peruanos / Banco Central de Reserva del Perú. 
PALMA, Ricardo

1961[1886] La bohemia de mi tiempo. En: Tradiciones peruanas completas. Edición y prólogo de Edith Palma. Con siete extensos apéndices y una selección de cartas del autor. 4ta. edición. Madrid: Aguilar: 1293-1321.

PÉREZ, Trinidad Manuel

1875[1862] La industria y el poder. Drama en tres actos. Lima: Imprenta Liberal de El Correo del Perú.

1872 "Nurerdín-Kan". El Correo del Perú. Año II, Núm. IV-XXIX.

1859 El emigrado español. Drama en cuatro actos. Lima: Tipografía de Aurelio Alfaro y Cía.

TAURO, Alberto

1976 Clorinda Matto de Turner y la novela indigenista. Lima: Universidad Nacional Mayor de San Marcos.

THOMPSON, E.P.

2012[1963] La formación de la clase obrera en Inglaterra. Prólogo de Antoni Domènech. Prefacio de Eric Hobsbawm. Madrid: Capitán Swing.

Zanutelli Rosas, Manuel

2006 Periodistas peruanos del siglo XIX. Itinerario biográfico. Lima: Universidad de San Martín de Porres. 